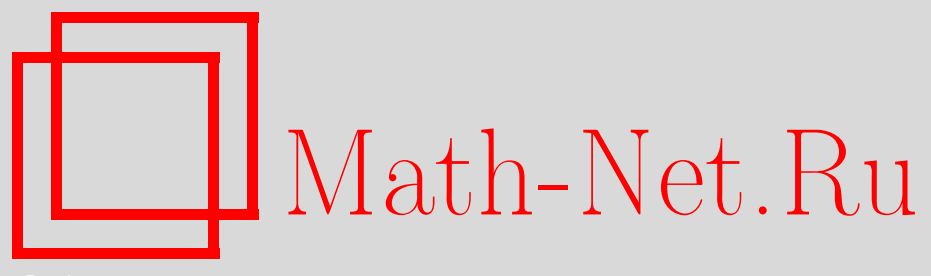

А. К. Кравцева, Бесконечномерные уравнения Шрёдингера с полиномиальными потенциалами и представление их решений в виде интегралов Фейнмана, Матем. заметки, 2013, том 94, выпуск 5, 788-791

DOI: https://doi.org/10.4213/mzm10254

Использование Общероссийского математического портала Math-Net.Ru подразумевает, что вы прочитали и согласны с пользовательским соглашением http://www.mathnet.ru/rus/agreement

Параметры загрузки:

IP : 54.89 .56 .158

26 апреля 2023 г., 14:55:31

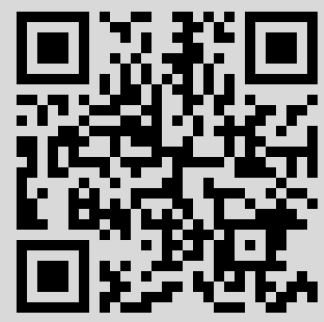




\section{Бесконечномерные уравнения Шрёдингера с полиномиальными потенциалами и представление их решений в виде интегралов Фейнмана}

\section{А. К. Кравцева}

1. Введение. В статье получены условия существования интегралов Фейнмана в смысле аналитического продолжения и найдены их представления в виде гауссовских интегралов. Описан класс эволюционных уравнений, обладающих решениями, представимыми с помощью интегралов Фейнмана. Ранее представления решений эволюционных уравнений в виде интегралов Фейнмана были получены в работах Альбеверио, Бутко, Неклюдова, Смолянова, Токарева, Трумена, Шавгулидзе, Шамарова [1]-[8] и многих других. В статье развивается подход работ Смолянова, Шавгулидзе [5]-[7], в которых фейнмановский интеграл определялся через аналитическое продолжение по параметру. В данной работе используется продолжение в пространстве операторов, благодаря чему удается заметно расширить класс эволюционных уравнений, которые имеют решения, описываемые интегралами Фейнмана.

2. Интегралы Фейнмана. Обозначим через $H$ вещественное сепарабельное гильбертово пространство со скалярным произведением $(\cdot, \cdot)$, порождающим норму $\|\cdot\|$. Пусть $e_{1}, e_{2}, \ldots, e_{n}, \ldots$ - ортонормированный базис пространства $H ; T$ - самосопряженный положительно-определенный ядерный оператор с собственными векторами $e_{1}, e_{2}, \ldots, e_{n}, \ldots$ и соответствующими собственными значениями $\lambda_{1}, \ldots, \lambda_{n}, \ldots ; P_{n}$ - ортогональный проектор, действующий из $H$ в $H_{n}$, где $H_{n}=\left\langle e_{1}, \ldots, e_{n}\right\rangle$. Через $X^{\mathbb{C}}$ обозначим комплексификацию произвольного гильбертова пространства $X$, через $B(X)$ - пространство линейных ограниченных операторов на данном пространстве. Пусть $\nu_{A}-$ семейство гауссовских мер с нулевыми средними и корреляционными операторами $A^{-1} T\left(A^{*}\right)^{-1}$, параметризованное обратимыми операторами $A \in B(H)$.

ОПРЕДЕЛЕНИЕ 1 . Функция $g: B\left(H^{\mathbb{C}}\right) \times \mathbb{C} \times H \rightarrow \mathbb{C}$ называется интегрируемой по мере Фейнмана, если в некоторой непустой области $V \subset B(H) \times B(H) \times \mathbb{R}$ функция

$$
G(A, B, \lambda)=\int_{H} g(B, \lambda, x) \nu_{A}(d x)
$$

определена, т.е. данный интеграл существует, и обладает аналитическим продолжением $\widetilde{G}(A, B, \lambda)$ в некоторую область $W \subset B\left(H^{\mathbb{C}}\right) \times B\left(H^{\mathbb{C}}\right) \times \mathbb{C}$. Величину $\widetilde{G}(A, B, \lambda)$ при комплексных $A, B, \lambda$ называют интегралом Фейнмана от функции $g$ и обозначают через

$$
\int_{H} g(B, \lambda, x) \exp \left\{-\frac{\left(T^{-1} A x, A x\right)}{2}\right\} d x .
$$

Пусть $q_{2 l}: H \times \cdots \times H \rightarrow \mathbb{R}-2 l$-линейная непрерывная симметричная форма, причем $l \geqslant 2$ и $q_{2 l}(x, \ldots, x)>0$ для любого $x$ из $H$, не равного нулю. Пусть, далее, $p(x)=$ $\left(q_{2 l}(x, \ldots, x)\right)^{1 /(2 l)}$. Предполагаем, что $p(x+y) \leqslant p(x)+p(y)$ для всех $x, y$, лежащих в $H$, и существует число $c>0$ такое, что неравенство

$$
\left|q_{2 l}\left(x_{1}, \ldots, x_{2 l}\right)\right| \leqslant c p\left(x_{1}\right) \cdots p\left(x_{2 l}\right)
$$

выполнено для всех $x_{1}, \ldots, x_{2 l} \in H$.

DOI: $10.4213 / \mathrm{mzm} 10254$ 
Введем пространство $F(H)$, состоящее из функций $f: H \rightarrow \mathbb{C}$, для каждой из которых существуют аналитическое продолжение $\tilde{f}: H^{\mathbb{C}} \rightarrow \mathbb{C}$ и константы $C_{1}>0, C_{2}>0,2 l \geqslant \varepsilon>0$ такие, что

$$
|\widetilde{f}(z)| \leqslant C_{1} \exp \left\{C_{2}|p(z)|^{2 l-\varepsilon}\right\}
$$

для всех $z$, лежащих в $H^{\mathbb{C}}$. Следующая теорема дает достаточное условие для существования функционального интеграла.

Tеорема 1. Пусть $A, C \in B\left(H^{\mathbb{C}}\right)$ - обратимъе операторы. Пусть существуют $B \in$ $B\left(H^{\mathbb{C}}\right), \lambda \in \mathbb{C}$, maкие, что $A C^{-1}=\lambda I+T B$, где $I-$ единичный оператор в $H,|\lambda|>$ $\|T\|\|B\|$. Пусть спектр $\sigma(A)$ оператора $A$ таков, что существует аналитическая кривая $\varphi:[0,1] \rightarrow \mathbb{C}$ такая, что $\varphi(t) \notin \sigma(A)$ для любого $t \in[0,1], \varphi(0)=0,|\varphi(1)|>\|A\|$, и пусть $f \in F(H)$. Тогда существует интеграл Фейнмана

$$
\int_{H} \tilde{f}(C x) \exp \left\{-(p(C x))^{2 l}\right\} \exp \left\{-\frac{1}{2}\left(T^{-1} A x, A x\right)\right\} d x .
$$

При этом найдутся оператор $S \in B(H)$ и константа $\omega>1$ такие, что данный интеграл равен гауссовскому интегралу

$$
\begin{aligned}
& \frac{1}{1+\operatorname{det}(I+(1 / \lambda) T B)} \\
& \int_{H} \tilde{f}\left(C A^{-1} x-\omega \frac{\|x\|^{2}}{(p(S x))^{2}} S x\right) \\
& \times \exp \left\{-\left(p\left(C A^{-1} x-\omega \frac{\|x\|^{2}}{(p(S x))^{2}} S x\right)\right)^{2 l}\right\} \\
& \times \exp \left\{\omega \frac{\|x\|^{2}}{(p(S x))^{2}}\left(T^{-1} A C^{-1} S x, x\right)-\frac{1}{2} \omega^{2} \frac{\|x\|^{4}}{(p(S x))^{4}}\left(T^{-1} A C^{-1} S x, A C^{-1} S x\right)\right\} \\
& \times J(x) \nu_{I}(d x),
\end{aligned}
$$

где

$$
\begin{aligned}
J(x)=\lim _{n \rightarrow \infty} \operatorname{det}(I- & \omega \frac{\left\|P_{n} x\right\|^{2}}{\left(p\left(S_{n} x\right)\right)^{2}} P_{n} A C^{-1} S_{n}-P_{n} A C^{-1} S_{n} x \otimes \frac{2 \omega}{\left(p\left(S_{n} x\right)\right)^{2}} P_{n} x \\
& \left.+P_{n} A C^{-1} S_{n} x \otimes \frac{2 \omega\left\|P_{n} x\right\|^{2}}{\left(p\left(S_{n} x\right)\right)^{2 l+2}} q_{2 l}\left(S_{n} x, \ldots, S_{n} x, S_{n}\right)\right) \quad \forall x \neq 0,
\end{aligned}
$$

$S_{n}=S P_{n}$

Схема ДоказАтельства. Будем рассматривать вещественные операторы $A, B, C \in$ $B(H)$ и положительное число $\lambda$ такие, что $A$ обратим, $\lambda>\|T\|\|B\|, C=(\lambda I+T B)^{-1} A$.

В интеграле (1) сделаем замену гауссовской меры; в результате получим

$$
\int_{H} f\left(C A^{-1} y\right) \exp \left\{-\left(p\left(C A^{-1} y\right)\right)^{2 l}\right\} \exp \left\{-\frac{1}{2}\left(T^{-1} y, y\right)\right\} d y .
$$

По свойству данной меры интеграл (3) равен

$$
\lim _{n \rightarrow \infty} \frac{1}{\sqrt{(2 \pi)^{n} \lambda_{1} \cdots \lambda_{n}}} \int_{H_{n}} f\left(C A^{-1} y\right) \exp \left\{-\left(p\left(C A^{-1} y\right)\right)^{2 l}-\frac{1}{2}\left(T^{-1} y, y\right)\right\} d y .
$$

K конечнократному интегралу применим преобразование

$$
y=x-\omega \frac{\|x\|^{2}}{(p(S x))^{2}} P_{n} A C^{-1} S x, \quad \text { где } \quad S e_{i}=\gamma_{i} e_{i}, \quad \gamma_{i} \searrow 0,
$$


снова перейдем к пределу при $n \rightarrow+\infty$ и получим равенство интегралов (1) и (2). При большом $\omega>1$ старший член

$$
\exp \left\{-\omega^{2 l}\left\{\frac{\|x\|^{4 l}}{(p(S x))^{2 l}}\right\}\right\}
$$

в экспоненте $\exp \left\{-\left(p\left(C A^{-1} x-\omega\left\{\|x\|^{2} /(p(S x))^{2}\right\} S x\right)\right)^{2 l}\right\}$ позволит доказать сходимость и аналитичность последнего интеграла при комплексных $A, B, \lambda$. Теперь существование интеграла Фейнмана следует из равенства этого интеграла исходному при вещественных параметрах. Теорема доказана.

3. Представление решений. Рассматривается задача Коши для эволюционного уравнения

$$
\begin{gathered}
\frac{\partial}{\partial t} u(t, q)=\frac{1}{2} \Delta_{A^{-1} T_{1}\left(A^{*}\right)^{-1}} u(t, q)+v(C q) u(t, q), \\
u(0, q)=u_{0}(C q),
\end{gathered}
$$

где $T_{1}$ - ядерный симметричный положительно-определенный оператор, заданный в гильбертовом пространстве $H_{1}$, оснащенном скалярным произведением $(\cdot, \cdot)_{1}$, порождающим норму $\|\cdot\|_{1}, u:[0, \infty) \times H_{1} \rightarrow \mathbb{C}-$ искомая функция, $A, C \in B\left(H_{1}^{\mathbb{C}}\right)$,

$$
\Delta_{A^{-1} T_{1}\left(A^{*}\right)^{-1}}=\sum_{i, j=1}^{\infty}\left(A^{-1} T_{1}\left(A^{*}\right)^{-1} l_{i}, l_{j}\right) \frac{\partial^{2}}{\partial q_{i} \partial q_{j}},
$$

$l_{1}, l_{2}, \ldots, l_{n}, \ldots$ - ортонормированный базис, состоящий из собственных векторов оператора $T_{1}$. Пусть $v_{k}: H_{1} \times \cdots \times H_{1} \rightarrow \mathbb{R}-$ линейное по каждой из $k$ переменных непрерывное симметричное отображение, $k=1, \ldots, 2 l, l \geqslant 2, v_{2 l}(q, \ldots, q)>0$ при $q \neq 0$. При этом

$$
r\left(q_{1}+q_{2}\right) \leqslant r\left(q_{1}\right)+r\left(q_{2}\right) \quad \forall q_{1}, q_{2} \in H_{1}, \quad \text { где } \quad r(q)=\left(v_{2 l}(q, \ldots, q)\right)^{1 /(2 l)} .
$$

Предположим также, что для некоторых $c_{k}>0$ выполнены неравенства

$$
\left|v_{k}\left(q_{1}, \ldots, q_{k}\right)\right| \leqslant c_{k} r\left(q_{1}\right) \cdots r\left(q_{k}\right), \quad k=1, \ldots, 2 l .
$$

Положим

$$
v(q)=\sum_{i=1}^{2 l} v_{i}(q, \ldots, q) .
$$

Пусть $t>0, E=\left\{x \in C\left([0, t], H_{1}\right): x(t)=0\right\}$. Обозначим через $\Phi\left(H_{1}\right)$ пространство функций $u_{0}: H_{1} \rightarrow \mathbb{C}$, для каждой из которых имеются аналитическое продолжение $\widetilde{u}_{0}:$ $H_{1}^{\mathbb{C}} \rightarrow \mathbb{C}$ и константы $c>0,1 /\left(4\left\|T_{1}\right\|\right)>\varepsilon>0$ такие, что

$$
\left|\widetilde{u}_{0}(z)\right| \leqslant c \exp \left\{\varepsilon\|z\|_{1}^{2}\right\} \quad \forall z \in H_{1}^{\mathbb{C}} .
$$

Teорема 2. Пусть $A, C$ - обратимые операторы из $B\left(H_{1}^{\mathbb{C}}\right)$. Существуют $\lambda \in \mathbb{C}, B \in$ $B\left(H_{1}^{\mathbb{C}}\right)$ mакие, что $A C^{-1}=\lambda I_{1}+T_{1} B$, где $I_{1}-$ единичный оператор в $H_{1},|\lambda|>\left\|T_{1}\right\|_{1}\|B\|_{1}$. Пусть спектр $\sigma(A)$ оператора $A$ таков, что найдется аналитическая кривая $\varphi:[0,1] \rightarrow \mathbb{C}$ такая, что $\varphi(t) \notin \sigma(A)$ для любого $t \in[0,1], \varphi(0)=0,|\varphi(1)|>\|A\|_{1}$, и пусть $u_{0} \in \Phi\left(H_{1}\right)$. Тогда задача Коши имеет решение, которое представляется в виде интеграла Фейнмана в смысле аналитического продолжения,

$$
\begin{aligned}
u(t, q)=\int_{E} \exp \{ & \left.\int_{0}^{t} v(C(q+x(\tau))) d \tau\right\} u_{0}(C(x(0)+q)) \\
& \times \exp \left\{-\frac{1}{2} \int_{0}^{t}\left(T_{1}^{-1} A x^{\prime}(\tau), A x^{\prime}(\tau)\right)_{1} d \tau\right\} d x .
\end{aligned}
$$


Схема ДоКАЗательства. Предположим, что $A$ и $C$ - произвольные вещественные обратимые операторы из $B\left(H_{1}\right)$. Тогда гауссовский интеграл $(6)$ является решением уравнения (4), удовлетворяющим начальному условию (5) [9]. При этом мера порождается квадратичной формой $b(x)=\int_{0}^{t}\left(T_{1}^{-1} A x^{\prime}(\tau), A x^{\prime}(\tau)\right)_{1} d \tau$, определенной на пространстве $E_{0}$, состоящем из функций $x \in E$ таких, что

- для любого $\tau \in[0, t] x(\tau) \in A^{-1} T_{1}^{1 / 2} H_{1}$;

- п.в. на $[0, t]$ существует $x^{\prime}(\tau) \in A^{-1} T_{1}^{1 / 2} H_{1}$;

- выполнено

$$
\int_{0}^{t}\left(T_{1}^{-1} A x^{\prime}(\tau), A x^{\prime}(\tau)\right)_{1} d \tau<\infty .
$$

Зафиксируем произвольное $0<\delta<1 / 2$. Рассмотрим элемент $\xi \in E$ и представим его в виде $\xi(\tau)=h(\tau)+e(\tau) \xi(0)$, где $e(\tau)=(t-\tau) / t$. Поскольку $h(0)=h(t)=0$,

$$
h(\tau)=\sum_{n=1}^{\infty} b_{n} \sin \left(\frac{\pi n \tau}{t}\right), \quad \text { где } \quad b_{n} \in H_{1}, \quad \tau \in[0, t] .
$$

Функции $h$, удовлетворяющие условию $\sum_{n=1}^{\infty} n^{2 \delta}\left\|b_{n}\right\|_{1}^{2}<\infty$, образуют гильбертово пространство $H_{\delta}$ со скалярным произведением $\left(h_{1}, h_{2}\right)_{\delta}=\sum_{n=1}^{\infty} n^{2 \delta}\left(b_{n}^{1}, b_{n}^{2}\right)_{1}$ для

$$
h_{i}(\tau)=\sum_{n=1}^{\infty} b_{n}^{i} \sin \left(\frac{\pi n \tau}{t}\right), \quad i=1,2, \quad \tau \in[0, t] .
$$

Интеграл $\int_{0}^{t}\left(T_{1}^{-1} h^{\prime}(\tau), h^{\prime}(\tau)\right)_{1} d \tau$ равен

$$
\frac{\pi^{2}}{2 t} \sum_{n=1}^{\infty} n^{2(1-\delta)} n^{2 \delta}\left(T_{1}^{-1} b_{n}, b_{n}\right)_{1} .
$$

Следовательно, он представляет собой квадратичную форму, порождающую гауссовскую меру в $H_{\delta}$ с ядерным корреляционным оператором. Из теоремы 1 вытекает существование функционального интеграла (6), если в качестве пространства $H$ взять $H_{\delta} \oplus H_{1}$. Равенство (4) продолжается по аналитичности на комплексные $A, B, C, \lambda$ такие, что $A C^{-1}=$ $\lambda I_{1}+T_{1} B$. При этом остается выполненным начальное условие (5). Теорема доказана.

В заключение автор выражает благодарность Е. Т. Шавгулидзе за постановку задачи, руководство работой и помощь в подготовке статьи.

\section{СПИСОК ЦИТИРОВАННОЙ ЛИТЕРАТУРЫ}

[1] С. Альбеверио, О. Г. Смолянов, Е. Т. Шавгулидзе, Докл. РАН, 361:6 (1998), 727-730. [2] Я. А. Бутко, Матем. заметки, 83:3 (2008), 333-349. [3] А. Ю. Неклюдов, Матем. заметки, 83:4 (2008), 581-589. [4] O. G. Smolyanov, A. G. Tokarev, A. Truman, J. Math. Phys., 43:10 (2002), 5161-5171. [5] О.Г. Смолянов, Е. Т. Шавгулидзе, Континуальные интеграль, Изд-во Моск. ун-та, М., 1990. [6] О. Г. Смолянов, Е. Т. Шавгулидзе, Докл. PAH, 390:3 (2003), 321-324. [7] О. Г. Смолянов, Е. Т. Шавгулидзе, Докл. PA H, 408:1 (2006), 28-33. [8] Н. Н. Шамаров, Матем. заметки, 75:2 (2004), 311-316. [9] Ю. Л. Далецкий, С.В. Фомин, Меры и дифференииальные уравнения в бесконечномерных пространствах, Наука, М., 1983.

\section{А. К. Кравцева}

Поступило

Московский государственный 25.04.2013 университет им. М. В. Ломоносова

E-mail: k-anna-k@mail.ru 